\title{
The health risks of ART
}

\author{
SSS Science \& Society Series on Sex and Science
}

\author{
Daria Grafodatskaya, Cheryl Cytrynbaum \& Rosanna Weksberg
}

A ssisted reproductive technology (ART) has become a standard intervention for couples with infertility problems, especially as ART is highly successful and overall carries low risks [1,2]. The number of infants born following ART has increased steadily worldwide, with more than 5,000,000 so far [3]. In industrialized countries, $1-4 \%$ of newborns have been conceived by using ART [4,5], probably owing to the fact that couples frequently delay childbearing until their late 30s, when fertility decreases in both men and women [2]. Considering the possibility that male fertility might be declining, as Richard Sharpe has discussed in this series [6], it is likely that ART will be even more widely used in the future. Yet, as the rate of ART and the total number of pregnancies has increased, it has become apparent that ART is associated with potential risks to the mother and fetus. The most commonly cited health problems pertain to multiple gestation pregnancies and multiple births. More recently, however, concerns about the risks of birth defects and genetic disorders have been raised. There are questions about whether the required manipulations and the artificial environments of gametes and embryos are potentially creating short- and long-term health risks in mothers and children by interfering with epigenetic reprogramming.

Notwithstanding, ART represents a tremendous achievement in human reproductive medicine. The birth of Louise Brown, the first 'test tube baby' in 1978, was the result of the collaborative work of embryologist Robert Edwards and gynaecologist Patrick Steptoe [7]. This success was a culmination of many years of work at universities and clinics worldwide. An initial lack of support, as well as criticism from ethicists and the church, delayed the opening of the first in vitro fertilization (IVF) clinic in Bourn Hall near Cambridge until 1980. By 1986, 1,000 children conceived by IVF at Bourn Hall had been born [8]. In 2010, Edwards received the Nobel Prize in Medicine for the development of IVF. Regrettably, Steptoe had passed away in 1988 and could not share the honour.

\section{...as the rate of $\mathrm{ART}$ and the total number of pregnancies has increased, it has become apparent that ART is associated with potential risks to mother and fetus}

Over the next decades, many improvements in IVF procedures were made to reduce the risks of adverse effects and increase success rates, including controlled ovarian stimulation, timed ovulation induction, ultrasound-guided egg retrieval, cryopreservation of embryos and intracytoplasmic sperm injection (ICSI)—a technique in which a single sperm cell is injected into an oocyte using a microneedle. In addition, there were further improvements such as assisted hatching and in media composition, such as sequential media, which allow the in vitro culture of the embryo to reach the blastocyst stage [8].

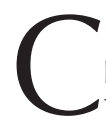

urrent IVF procedures involve multiple steps including ovarian stimulation and monitoring, oocyte retrieval from the ovary, fertilization in vitro and embryo transfer to the womb. Whereas the first IVF cycles, including the conception of Louise Brown, used natural ovulatory cycles, which result in the retrieval of one or two oocytes, most IVF cycles performed

today rely on controlled ovarian stimulation using injectable gonadotropinsfollicle stimulating hormone and luteinizing hormone-in supraphysiological concentrations for 10-14 days, followed by injection of human chorionic gonadotropin (hCG) 38-40 h before egg retrieval to trigger ovulation. This updated protocol makes it possible to grow multiple follicles and to retrieve 10-20 oocytes in one IVF cycle, thereby increasing the number of eggs available for fertilization.

Post-retrieval, the embryologist places an egg and sperm together in a test tube for fertilization. Alternatively, a single sperm cell can be injected into an egg by using ICSI. This procedure was initially developed for couples with poor sperm quality [9], but has become the predominant fertilization technique used in many IVF clinics worldwide [8]. The developing embryos are monitored by microscopy, and viable embryos are transferred into the woman's womb for implantation. Louise Brown, as with many embryos today, was transferred three days after egg retrieval, at approximately the eight-cell stage. However, using sequential media, many clinics advocate culturing embryos until day five when they reach

SSS

Science \& Society Series on Sex and Science

Sex is the greatest invention of all time: not only has sexual reproduction facilitated the evolution of higher life forms, it has had a profound influence on human history, culture and society. This series explores our attempts to understand the influence of sex in the natural world, and the biological, medical and cultural aspects of sexual reproduction, gender and sexual pleasure. 
the blastocyst stage. The prolonged culture period allows self-selection of the most viable embryos for transfer and increases the chance of a viable pregnancy. Excess embryos can be cryopreserved and transferred at a later date by using a procedure known as frozen embryo transfer (FET). In this article we use the term ART to refer to IVF procedures with or without ICSI and FET.

Embryos can also be screened for chromosomal aneuploidies-missing or extra chromosomes-by preimplantation genetic diagnosis (PGD) when indicated and when available. PGD can also be used to test fertile couples at increased risk of genetic disorders. To perform PGD, a single cell is obtained from three-day-old embryos for molecular testing, for example sequencing for inherited monogenic disorders or fluorescent in situ hybridization for chromosomal abnormalities [8]. Only embryos with a normal chromosomal constitution, and without the genetic disorder in question, would then be transferred into the woman's womb.

$\mathrm{D}$ espite tremendous progress during the past three decades, people undertaking ART still face a considerable risk of failure to achieve parenthood. The rate of clinical pregnancies in Bourn Hall between 1980 and 1985 was 24\% and $14 \%$ in women younger and older than 40 years, respectively [10]. The reported rates for clinical pregnancies and live births vary by country; the average delivery rate is $22.4 \%, 23.3 \%$ and $17.1 \%$ for IVF, ICSI and FET cycles, respectively [11]. According to the last Centers for Disease Control and Prevention report in 2009, the average livebirth rate was $35 \%$ per fresh ART cycle, although it sharply declines with age, from $45 \%$ among women younger than 35 years to $7 \%$ among women older than 42 years [5]. The reasons include poor response to ovarian stimulation, ovarian hyperstimulation syndrome and failure of eggs to fertilize. However, these failures occur in only a minority of patients and the success rate of egg retrieval and fertilization leading to embryo transfer is a remarkable $90 \%$ [12].

Implantation remains the least understood process and is a key rate-limiting step in ART. Poor embryo quality is considered to be the main cause of implantation failure and it reflects a high incidence of chromosomal aneuploidies, which increases with maternal age [13]. One obvious solution to improve implantation rates is to transfer more embryos. However, this also increases the risk of multiple births, and related morbidity and mortality in newborns. An alternative approach is to select for good-quality embryos by culturing them to the blastocyst stage, because it seems that aneuploid embryos arrest by this stage and that blactocysts are more likely to have a normal chromosomal complement. There is ongoing research aimed at identifying viable embryos through PGD and metabolic profiling [13].

\section{Despite tremendous progress during the past three decades, people undertaking ART still face a considerable risk of failure to achieve parenthood}

It has also been suggested that failure to implant could be caused by the inability of the embryo to hatch out of a glycoprotein layer surrounding the embryo, known as the 'zona pellucida'; this layer hardens if the embryo is cultured or frozen. Assisted hatching by rupturing the zona pellucida before embryo transfer does increase clinical pregnancy rates, especially for thawed embryos [13]. Another factor linked to the failure of implantation is endometrial receptivity. The endometrium consists of multi-layered mucosa cells in the inner wall of the uterus, which undergoes coordinated remodelling during the menstrual cycle and there is a specific time window when it is receptive to embryo implantation. Several research studies have identified molecular biomarkers of poor endometrial receptivity, showing that prostaglandins, cell adhesion molecules, mucins and cytokines are important [13].

W hen it comes to health risks for mothers and infants, the use of ART increases the risk of multiple births, including higher rates of caesarian sections, prematurity, low birth weight, infant death and disability. More recently, concerns regarding elevated risks of birth defects, genetic abnormalities, neurodevelopmental disorders and imprinting disorders have been reported; however, not all are substantiated. There are still many unanswered questions regarding the potential shortand long-term health risks of ART for women and children, and there are tremendous challenges in studying the safety of ART procedures. Apart from the subset of individuals undergoing ART for social reasons-single parents or same sex couples-most patients are subfertile couples. Subfertility, defined as a failure to conceive naturally after 12 months of unprotected intercourse, affects $8-20 \%$ of couples [2], and it can occur for a variety of unknown or known reasons including maternal factors-endocrine, hormonal, endometriosis and blocked fallopian tubes-and paternal factors such as spermatogenesis abnormalities.

Most studies have assessed the risks of ART by comparing the outcomes of ART-conceived pregnancies to naturally conceived pregnancies. There is emerging evidence that underlying maternal or paternal subfertility might be an important factor in obstetric, neonatal and childhood outcomes in the ART population. Therefore, to determine the specific health risks associated with the ART process itself, the outcomes of ART-conceived pregnancies should be assessed in comparison with naturally conceived pregnancies in subfertile parents, which is methodologically difficult. Alternatively, studying the health risks of ART in fertile couples-for instance, same-sex couples and couples at risk of genetic disorders-would be informative, but the number of such couples is relatively small.

W omen who undergo ART are at risk of ovarian hyperstimulation syndrome (OHSS). OHSS is a complication of ovulation induction resulting in enlargement of ovaries and retention of fluids leading to various secondary complications, which normally resolve within two weeks, but can persist if pregnancy occurs. Patients with OHSS can be offered embryo cryopreservation and frozen embryo transfer when symptoms resolve. Moderate forms of OHSS occur in $5 \%$ of patients undergoing ART; $2 \%$ of patients require hospitalization. Death occurs with an incidence of approximately 3 per 100,000 ART cycles [14]. OHSS is predominantly caused by human chorionic gonadotropin injection used for inducing final oocyte maturation and ovulation. Research is focused on optimizing alternative stimulation protocols [14].

The use of supraphysiological concentrations of hormones during ovarian stimulation has also raised concerns that ART can increase cancer risks linked to hormonal fluctuations. These include breast, ovarian, endometrial, cervical and colon cancers, 
as well as melanoma. Studies evaluating the risks of cervical cancers, colon cancers and melanoma have not demonstrated increased risks for women undergoing ART [1]. The data for breast, ovarian and endometrial cancer is more complex, however, and more research is required to conclusively determine whether there is an increased risk.

$\mathrm{T}$ he perinatal and obstetric risks of ART are most significantly influenced by multiple pregnancies. These are at a more than $60 \%$ risk of low birth weight or premature delivery [2], and related risks of pregnancy complications such as gestational diabetes, abnormal placentation and hypertensive disorders [1]. Multiple pregnancies occur in $1 \%$ of naturally conceived pregnancies and $25-50 \%$ of ART pregnancies, owing to multiple embryo transfer. In the Western world, about $30-50 \%$ of all twin pregnancies result from ART [2]. Whilst double or triple embryo transfer is still common, the development of cryopreservation techniques and extended blastocyst culture has increased the use of single embryo transfer (SET), especially for younger women. Many European countries and the province of Quebec, in Canada, where ART is publicly funded, have adopted a policy of SET, which has dramatically decreased the incidence of multiple pregnancies. In Belgium and Quebec, SET policies have reduced multiple pregnancies from $19 \%$ to $3 \%$ and from $27 \%$ to $6 \%$, respectively. It has been argued that SET results in a lower live-birth rate than a double-embryo transfer, but this is almost completely overcome by an additional single frozen embryo cycle [2]

\section{...there are tremendous challenges in studying the safety of ART procedures}

The question of whether ART increases the risks of pregnancy complications, including prematurity and low birth weight in singletons, remains unresolved; several studies have found an increased risk, but others have not replicated these findings $[1,2]$. It has been suggested that the fertility history of patients undergoing ART is an important factor, as there is an association between the length of time to conception and prematurity and birth weight [15]. Prematurity and low birth weight are also known to be associated with long-term health effects, including adult onset coronary artery disease, hypertension, obesity and type 2 diabetes [16,17].

Various studies have also reported a higher incidence of congenital anomalies in ART-conceived children, with a suggested 30\% increase of malformations [2]. However, this is another risk that might be attributable to parental subfertility, as a study comparing children conceived by ART to subfertile parents and children conceived naturally to subfertile parents did not find any significant difference in the congenital anomaly rate [2]. Findings from another study of the risks of birth defects in children conceived naturally to women with and without a history of subfertility compared with children conceived with the assistance of ART also suggest that it is subfertility, rather than ART, that is associated with an increased risk of birth defects [18].

S everal studies reported an increased risk of cerebral palsy and other neurological abnormalities in children conceived by ART [2]. But again, these findings are mainly attributed to complications resulting from multiple pregnancies including prematurity and low birth weight. The increased utilization of SET is therefore expected to result in fewer multiple pregnancies, which should result in a concomitant decrease in neurological complications. Further evidence that neurological complications in ART children are not exclusively related to ART came from studies that have assessed neurodevelopmental outcomes, such as locomotion, cognition, language and behavioural development of ART children in comparison with naturally conceived children. These analyses did not reveal any differences when adjusted for confounding factors of low birth weight and prematurity. In a similar vein, numerous studies have investigated whether there is an increased incidence of autism in ART-conceived children, but these have been inconclusive [19].

There are potential concerns regarding the fertility of ART children. However, this requires future studies as most of this population is younger than 30 years of age. There is some evidence that boys conceived through ICSI have an increased rate of genital anomalies [2] and that males with severe infertility, such as low sperm counts, are more likely to carry chromosomal abnormalities, which could be passed on to their children conceived through ICSI [15].

It has also been suggested that there might be an increased risk of cancers in ART-conceived offspring. Although multiple studies have identified no such risk, a large Swedish study reported a marginally increased risk of cancer, including haematologic, eye, nervous system, solid tumours and histiocytosis [2]. Similarly to other ARTrelated adverse health outcomes, it has been suggested that the increased risk of cancer could be attributed to prematurity, a recognized risk factor for cancer, rather than to the ART procedure itself. Further long-term studies are required to determine if there is truly an increased risk of adult cancers in ART offspring.

\section{...there remain unanswered questions about both the health risks associated with $\mathrm{ART}$ and the potential mechanisms that could account for these findings}

One thing is clear from the available evidence to date: there remain unanswered questions about both the health risks associated with ART and the potential mechanisms that could account for these findings. One possible explanation is that the exposure of gametes and preimplantation embryos to the various steps of ART might affect growth and development of offspring through dysregulation of epigenetic pathways [20]. In addition, there is evidence that genetic and epigenetic alterations might be inherited from the gametes of subfertile parents, which would reinforce assertions that subfertility itself might play a role in ART-related health outcomes $[1,20]$.

$\amalg$ pigenetics refers to heritable changes in gene expression without alterations to the underlying DNA sequence. DNA methylation and modifications of histones are epigenetic modifications that determine active against repressive conformation of chromatin structure, thereby regulating gene expression and driving essential processes such as embryonic development, fetal organ development, cell differentiation and tissue-specific gene expression [21]. Genomic imprinting is a type of epigenetic gene regulation that uses epigenetic marks to silence specifically one of the parental alleles. There are 

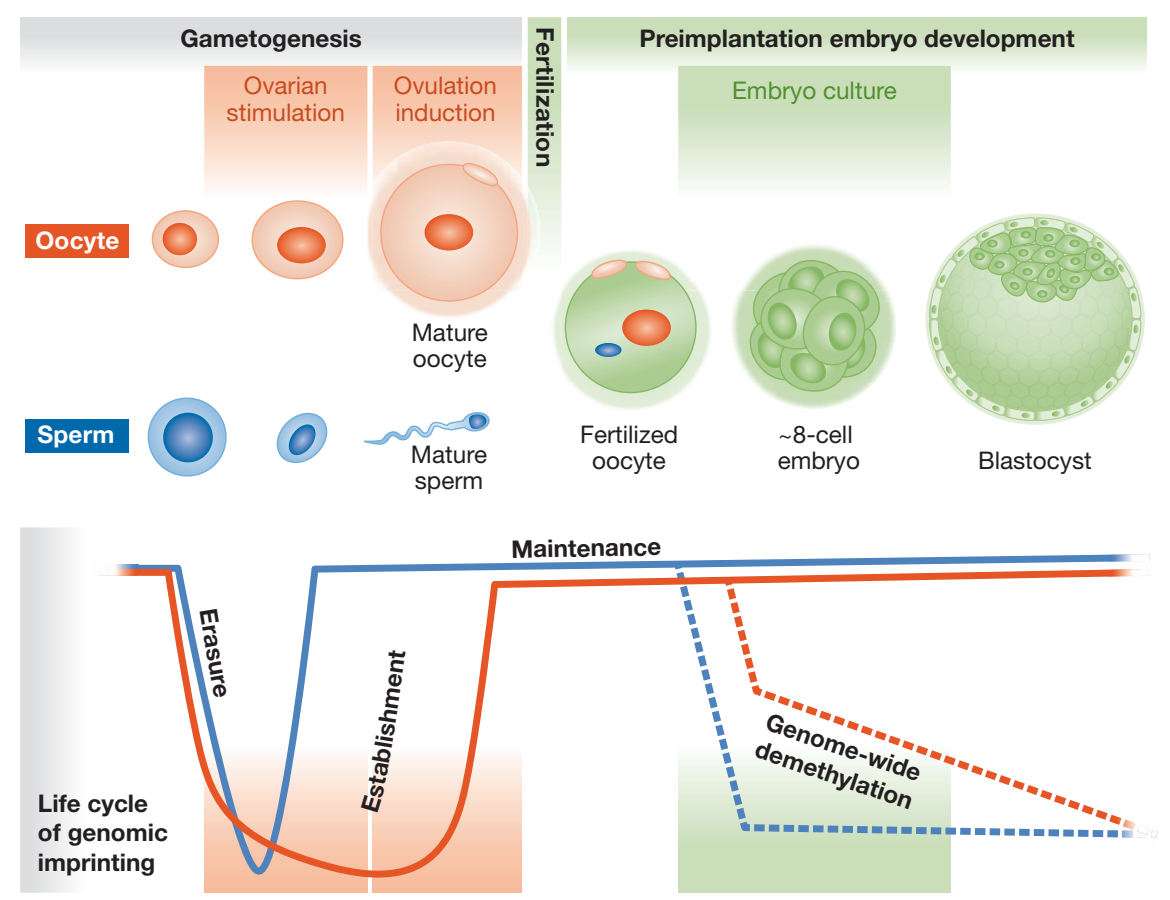

Fig 1 | Life cycle of genomic imprinting and assisted reproductive technology. Erasure, re-establishment and maintenance of genomic imprinting occur during gametogenesis and preimplantation embryo development. Blue and red solid lines show paternal and maternal methylation at imprinting centres through gametogenesis and early stages of preimplantation development. Imprinting marks are erased at early stages of gametogenesis. Re-establishment of imprinting occurs throughout gametogenesis, but finishes much later in oocytes compared with sperm. During preimplantation development, both maternal and paternal imprinting marks are maintained whilst the rest of the genome is demethylated. The paternal genome is demethylated rapidly and actively (dashed blue line) whilst the maternal genome is demethylated at a slower rate passively through cell division (dashed red line). Various steps of assisted reproductive technology such as ovarian stimulation, ovulation induction, gamete and embryo manipulation and culturing create unusual environments for gametes and embryos and thus, can interfere with proper establishment of imprinting marks in oocytes or maintenance of imprinting marks in embryos. Subfertility can be associated with epigenetic errors in imprinting erasure and/or establishment in both oocytes and sperm. Adapted from [23].

approximately 100 known imprinted genes in humans [22]. Most imprinted genes are found in clusters across the genome and are regulated by parent-specific DNA methylation and histone modification marks at cis-acting imprinting centres, as well as non-coding RNAs. Most of the known imprinted genes have functions related to growth and behaviour; disruption of the normally programmed parental expression of imprinted genes can therefore result in disorders related to growth and neurodevelopment.

Gametogenesis and embryogenesis are important stages of mammalian development that require genome-wide epigenetic reprogramming. During spermatogenesis, protamines replace most histone proteins to create a highly compacted DNA.
Establishment of DNA methylation imprints at paternally methylated imprinting centres is complete in males at the time of birth. In females, the establishment of maternally methylated imprinting centres begins during puberty and is almost complete in ovulated oocytes. After fertilization, the paternal genome undergoes rapid active DNA demethylation in which protamines are replaced by histones, whilst the maternal genome is passively demethylated, so that DNA methylation patterns are lost through cell divisions. Although, the whole genome undergoes demethylation, parentspecific DNA methylation is maintained at imprinting centres. Subsequently, the genome is remethylated and cell-typespecific epigenetic patterns are established as embryonic development proceeds.
The parent-specific DNA methylation at imprinting centres is maintained in somatic cells, but it is erased and re-established in the gametes starting a new cycle of imprinting (Fig 1; [23]). As the establishment and maintenance of imprinting marks coincides in timing with important stages of ART, such as oocyte maturation under supraphysiological hormone concentrations and embryo culture, it has been proposed that ART can lead to imprinting errors [24].

n 2001, the first evidence that genomic imprinting can be perturbed during ART procedures came from studying sheep fetuses derived from in vitro cultured embryos that presented with large offspring syndrome (LOS; [25]). LOS occurs sporadically in cattle and sheep conceived by IVF and is characterized by a $20-30 \%$ increase in birth weight frequently accompanied by congenital anomalies and placental dysfunction [24]. Owing to phenotypic similarities of LOS to the human overgrowth disorder Beckwith-Wiedemann syndrome (BWS), which is caused by the dysregulation of gene expression within an imprinted cluster on chromosome $11 \mathrm{p} 15.5$, the authors hypothesized that genes from the orthologous cluster in sheep or a closely related pathway could be dysregulated in LOS. They tested expression of the insulin-like growth factor 2 (IGF2) gene known to be overexpressed in BWS, and the IGF2R receptor gene, which is involved in clearance of IGF2 from the circulation. IGF2R is imprinted in sheep but not in humans. In sheep with LOS, no differences for IGF2 were found, but reduced expression of IGF2R was observed after loss of DNA methylation at the imprinting centre for this gene [25].

In the following decade, several studies provided further evidence that children conceived by ART might be at increased risk of imprinting disorders. The strongest case has been made for BWS and Angelman syndrome. BWS is the most common human overgrowth syndrome characterized by prenatal and postnatal overgrowth, congenital anomalies and tumour predisposition [26]. Angelman syndrome is a neurodevelopmental disorder characterized by microcephaly, severe intellectual disability and a unique behavioural profile including frequent laughter, smiling and excitability [27]. Multiple case reports from various countries indicate 

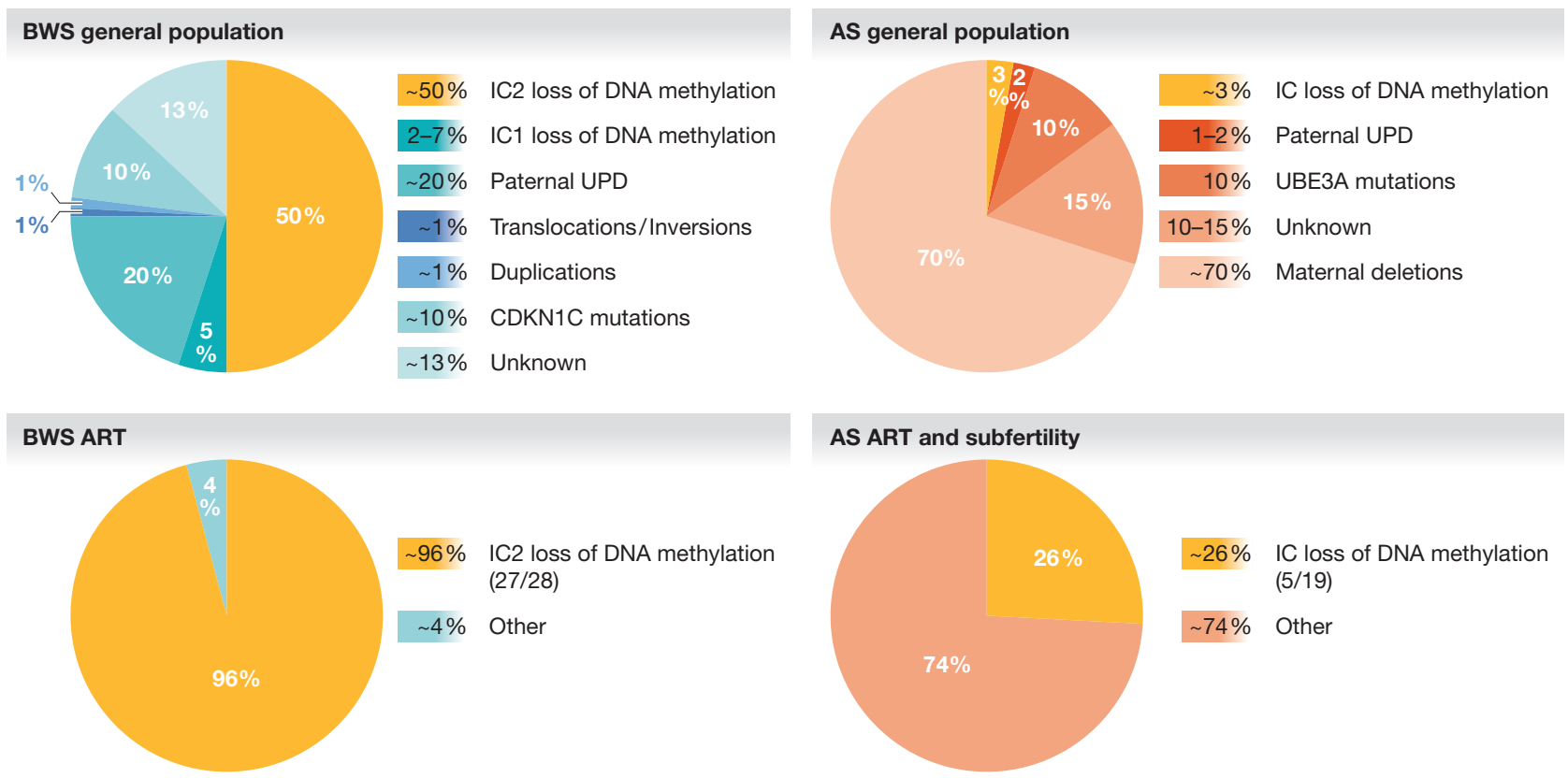

Fig 2 | Enrichment of epigenetic alterations in Beckwith-Wiedemann syndrome and Angelman syndrome after assisted reproductive technology. Loss of methylation (LOM) at imprinting centre 2 (IC2) on chromosome $11 \mathrm{p} 15.5$ contributes to $50 \%$ of Beckwith-Wiedemann syndrome (BWS) cases in the general population, whereas LOM at IC2 is found in 27 out of 28 cases (96\%) in the BWS assisted reproductive technology (ART) population, which represents a 1.9fold enrichment of this epigenetic defect. For Angelman syndrome (AS), methylation disruption at the 15q11-q13 imprinting centre contributes to 3\% of AS cases, and in the AS ART and subfertility population it was found in 5 out of 19 cases (26\%; eight fold enrichment). Data from the following publications were used for these calculations, BWS [31-35]; AS [35-36].

an increased frequency of BWS and Angelman syndrome in ART children (3-10-fold) compared with the general population. However, two cohort studies failed to replicate this association [28]. The low incidence of both BWS (1 in 13,700) and Angelman syndrome (1 in 15,000) in the general population [28] makes epidemiological studies difficult-the two cohort studies reported 2,492 and 6,052 ART children, respectively, and are probably underpowered to detect an increased risk of BWS and Angelman syndrome. However, even if there might be increased relative risks for these syndromes in ART children, the absolute risks in this population remain low.

$\mathrm{T}$ he molecular causes of BWS and Angelman syndrome are heterogeneous. They include genomic (deletion, uniparental disomy and gene mutation) and epigenetic (loss of imprinting due to aberrant DNA methylation) alterations at imprinted gene clusters on chromosomes $11 \mathrm{p} 5.5$ and $15 q 11-q 13$, respectively. These alterations occur with specific frequencies for each of the two disorders [26,27]. Results of molecular testing in children with these syndromes and conceived using ART, reveal an excess of epigenetic compared with genetic molecular alterations. For example, loss of DNA methylation at imprinting centre 2 occurs in about $50 \%$ of BWS cases in the general population, whereas several studies found loss of DNA methylation at imprinting centre 2 in 96\% (27/28) of BWS ART-conceived children. In Angelman syndrome, approximately $3 \%$ of cases in the general population have loss of methylation at $15 q 11-13$, whereas 5 out of 19 (26\%) Angelman syndrome children conceived by ART or naturally by parents with a history of subfertility had loss of DNA methylation at 15q11-13 (Fig 2).

The data for loss of DNA methylation in Angelman syndrome cases conceived naturally by subfertile parents highlights the fact that epigenetic alterations could, at least in part, result from underlying parental subfertility. Indeed, several studies have shown that abnormalities of spermatogenesis, such as oligospermia (low sperm concentration), low sperm motility or abnormal sperm morphology are associated with altered DNA methylation at imprinted loci. These occur in both maternal and paternal alleles of imprinting centres in sperm and could be transmitted to offspring conceived by ART [26]. One study of chromosomally normal fetuses spontaneously aborted at six to nine weeks of gestation found that DNA methylation alterations at imprinted loci were sometimes inherited from sperm. Thus, it is possible that this dysregulation of imprinting in male gametes might be one cause of the association between imprinting disorders and ART.

Studies of other known imprinted syndromes, such as Prader-Willi syndrome, Russell-Silver syndrome, maternal and paternal uniparental disomy of chromosome 14, pseudohypoparathyroidism type $1 \mathrm{~b}$ and transient neonatal diabetes mellitus, have either not demonstrated an association with ART or have been inconclusive owing to their small size [29]. A link has also been suggested between ART and the newly defined 'multiple maternal hypomethylation syndrome', which clinically presents either as BWS or transient neonatal diabetes mellitus, and is associated with loss of DNA methylation at multiple maternally methylated imprinting centres; loss of methylation at paternal imprinting centres has not been reported so far. Thus, human imprinting disorders that have been observed with increased relative frequency in ART offspring are confined 
to loss of DNA methylation at maternally methylated imprinting centres, similar to epimutations of IGF2R in LOS. One could propose that ART has a greater impact on female than male gametes, as the eggs are subjected to more environmental exposures-supraphysiological doses of hormones-and more manipulation than the sperm. However, studies of mouse in vitro cultured embryos and ART-exposed human and mouse gametes suggest that ART can also be associated with either loss or gain of DNA methylation on both maternal and paternal alleles [23].

M ouse models are a valuable method to investigate which stages of ART procedures can disrupt normal imprinting patterns. The advantage of using mouse models is the ability to investigate each of the parameters of ART-ovulation stimulation and embryo culturing - separately and at different stages of development. Furthermore, mouse models allow investigators to alter ART parameters, such as concentration of hormones or media for embryo culturing. Most importantly, studies in animal models have shown that ART procedures without the confounding factor of subfertility do have a negative impact on imprint regulation [23].

The exposure of maturing oocytes from mice to abnormally high doses of gonadotropins has been suggested to alter imprint establishment. Yet, studies performed directly on superovulated oocytes are inconclusive, as not all of them have demonstrated increased rates of DNA methylation errors at imprint centres compared with spontaneously ovulated oocytes. Interestingly, studies of DNA methylation in mouse blastocysts harvested from superovulated mothers identified an increased rate of DNA methylation errors at imprint centres. This included loss of DNA methylation at the paternally methylated H19-the imprinting centre on human chromosome 11 and mouse chromosome 7 implicated in BWS and the related undergrowth Russell-Silver syndrome. It suggests that superovulation also impairs imprinting maintenance;

As the establishment and maintenance of imprinting marks coincides in timing with important stages of ART [...] it has been proposed that ART can lead to imprinting errors probably by affecting the ability of the oocyte to synthesize and store sufficient maternal factors (RNA and proteins; [23]). In support of this hypothesis, four maternal effect proteins have been previously identified that are involved in imprinting maintenance in preimplantation embryos. It was also found that imprint errors arise in blastocysts in a dose-dependent mannerhigher doses of hormones resulted in DNA methylation errors in a larger number of embryos [23].

Another factor that might contribute to imprinting errors is the micromanipulation of gametes during IVF and ICSI procedures. Evidence supporting this hypothesis includes the observation in mouse models that a higher number of IVF embryosresulting from superovulation alone or superovulation and embryo culturinghave aberrant H19 DNA methylation compared with in vivo conceived embryos [23]. Media with varying compositions are used in ART clinics, and whilst all of the media are suboptimal for normal maintenance of all DNA imprints in mouse embryos, the number of embryos with aberrant DNA methylation at imprinting centres varies depending on the media [23]. Interestingly, it was also found that embryos with faster rates of development are more prone to loss of DNA methylation at imprinting centres [23].

T hough it is not yet clear how these findings relate to ART in humans, the mouse research is crucial for informing human studies about which variables should be addressed to optimize the safety and efficacy of ART procedures. Apart from ART itself, it has been shown that compromised fertility in mice results in loss or delay of DNA methylation acquisition in one of three tested imprinted genes. The compromised fertility is induced by genetic manipulation of a gene involved in communication between oocytes and surrounding follicular cells, which is crucial for proper oocyte maturation. The results suggest that the observed loss of DNA methylation could be caused by impaired transport of metabolites from follicular cells to oocytes, which is important for imprint establishment [23].

Data linking dysregulation of imprinted loci and ART is limited to several imprinted gene clusters associated with clinically recognizable syndromes. However, there are more genes in the human genome that have been discovered to be, or are predicted to be, imprinted [22] but are not yet known to be associated with clinical phenotypes. Potentially, ART can lead to dysregulation of these imprinted genes, which might be another, as yet unrecognized factor contributing to neonatal and long-term health problems of ART-conceived children. At this point, it is also not clear whether epigenetic disruption during ART is limited to imprinted genes or has more global effects on the genome. The data for genome-wide DNA methylation analysis are limited in both human and mouse to individuals with no apparent disease phenotype. So far, these data have been inconclusive $[23,28]$.

\section{One could propose that ART has a greater impact on female than male gametes, as the eggs are subjected to more environmental exposures $[. .$.$] and more$ manipulation than the sperm}

Despite significant advances in the efficacy and success of ART procedures during the past few decades, the health risks, especially related to long-term outcomes in ART-conceived children, remain poorly understood. Moreover, the phenomena known as 'fetal programming' when maternal and in utero exposures can lead to various adult onset disease susceptibilities-have been suggested to be transmissible to the next generations, probably through epigenetic mechanisms [30]. In the case of ART procedures, the effect of 'unusual' environments during gametogenesis and early embryonic development on adult-onset disease and trans-generational inheritance is still not clear. Additional research is needed to elucidate the effects of ART on genomewide epigenetic patterns and their link to human disease. As ART will continue to be an important medical intervention and the number of children born with the help of ART procedures will probably continue to rise in the future, it is crucial to understand the associated health risks and underlying molecular mechanisms of these technologies. This will increase the safety of this intervention and enable couples using ART to be fully informed regarding both present and future health-related risks. 


\section{CONFLICT OF INTEREST}

The authors declare that they have no conflict of interest.

\section{REFERENCES}

1. Metwally M, Ledger WL (2011) Long-term complications of assisted reproductive technologies. Hum Fertil (Camb) 14: 77-87

2. Savage T, Peek J, Hofman PL, Cutfield WS (2011) Childhood outcomes of assisted reproductive technology. Hum Reprod 26: 2392-2400

3. Adamson DG, Zegers-Hochschild F, Ishihara O, Sullivan E, Mansour R, Nygren K, Banker M, Dyer S, de Mouzon J (2012) ICMART World Report: Preliminary 2008 Data. London, UK: European Society for Human Reproduction and Embryology Annual Meeting

4. de Mouzon J, Goossens V, Bhattacharya S, Castilla JA, Ferraretti AP, Korsak V, Kupka M, Nygren KG, Nyboe Andersen A (2010) Assisted reproductive technology in Europe, 2006: results generated from European registers by ESHRE. Hum Reprod 25: 1851-1862

5. CDC (2009) 2009 assisted reproductive technology report. http://www.cdc.gov/ART/ ART2009/

6. Sharpe RM (2012) Sperm counts and fertility in men: a rocky road ahead. EMBO Rep 13: 398-403

7. Edwards RG (2001) The bumpy road to human in vitro fertilization. Nat Med 7: 1091-1094

8. Zhao Y, Brezina P, Hsu CC, Garcia J,

Brinsden PR, Wallach E (2011) In vitro fertilization: four decades of reflections and promises. Biochim Biophys Acta 1810: 843-852

9. Palermo G, Joris $H$, Devroey $P$, Van Steirteghem AC (1992) Pregnancies after intracytoplasmic injection of single spermatozoon into an oocyte. Lancet $\mathbf{3 4 0}$ 17-18

10. Steptoe PC, Edwards RG, Walters DE (1986) Observations on 767 clinical pregnancies and 500 births after human in-vitro fertilization. Hum Reprod 1: 89-94

11. Nygren KG, Sullivan E, Zegers-Hochschild F, Mansour R, Ishihara O, Adamson GD de Mouzon J (2011) International Committee for Monitoring Assisted Reproductive Technology (ICMART) world report: assisted reproductive technology 2003. Fertil Steril 95: 2209-2222

12. Fasouliotis SJ, Schenker JG (2003) Failures in assisted reproductive technology: an overview. Eur J Obstet Gynecol Reprod Biol 107: 4-18

13. KootYE, Teklenburg G, Salker MS, Brosens IJ, Macklon NS (2012) Molecular aspects of implantation failure. Biochim Biophys Acta 1822: 1943-1950
14. Devroey P, Polyzos NP, Blockeel C (2011) An OHSS-free clinic by segmentation of IVF treatment. Hum Reprod 26: 2593-2597

15. Dupont C, Sifer C (2012) A review of outcome data concerning children born following assisted reproductive technologies. ISRN Obstet Gynecol 2012: 405382

16. Barker DJ (2004) The developmental origins of chronic adult disease. Acta Paediatr Suppl 93: $26-33$

17. Moster D, Lie RT, Markestad T (2008) Longterm medical and social consequences of preterm birth. N Engl / Med 359: 262-273

18. Davies MJ, Moore VM, Willson KJ, Van Essen P, Priest K, Scott H, Haan EA, Chan A (2012) Reproductive technologies and the risk of birth defects. N Engl J Med 366: 1803-1813

19. Hvidtjorn D, Schieve L, Schendel D, Jacobsson B, Svaerke C, Thorsen P (2009) Cerebral palsy, autism spectrum disorders, and developmental delay in children born after assisted conception: a systematic review and meta-analysis. Arch Pediatr Adolesc Med 163: $72-83$

20. Paoloni-Giacobino A (2007) Epigenetics in reproductive medicine. Pediatr Res 61: 51R-57R

21. Portela A, Esteller M (2010) Epigenetic modifications and human disease. Nat Biotechnol 28: 1057-1068

22. Choufani S et al (2011) A novel approach identifies new differentially methylated regions (DMRs) associated with imprinted genes. Genome Res 21: 465-476

23. Denomme MM, Mann MR (2012 Genomic imprints as a model for the analysis of epigenetic stability during ARTs. Reproduction 144: 393-409

24. Sinclair KD, Young LE, Wilmut I, McEvoy TG (2000) In-utero overgrowth in ruminants following embryo culture: lessons from mice and a warning to men. Hum Reprod 5 (Suppl): 68-86

25. Young LE et al (2001) Epigenetic change in IGF2R is associated with fetal overgrowth after sheep embryo culture. Nat Genet 27: 153-154

26. Choufani S, Shuman C, Weksberg R (2010) Beckwith-Wiedemann syndrome. Am J Med Genet C Semin Med Genet 154C: 343-354

27. Buiting K (2010) Prader-Willi syndrome and Angelman syndrome. Am / Med Genet C Semin Med Cenet 154C: 365-376

28. Odom LN, Segars J (2010) Imprinting disorders and assisted reproductive technology. Curr Opin Endocrinol Diabetes Obes 17: 517-522

29. Amor DJ, Halliday J (2008) A review of known imprinting syndromes and their association with assisted reproduction technologies. Hum Reprod 23: 2826-2834

30. Drake AJ, Seckl JR (2011) Transmission of programming effects across generations. Pediatr Endocrinol Rev 9: 566-578
31. DeBaun MR, Niemitz EL, Feinberg AP (2003) Association of in vitro fertilization with Beckwith-Wiedemann syndrome and epigenetic alterations of LIT1 and H19. Am J Hum Genet 72: 156-160

32. Doornbos ME, Maas SM, McDonnell J, Vermeiden JP, Hennekam RC (2007) Infertility, assisted reproduction technologies and imprinting disturbances: a Dutch study. Hum Reprod 22: 2476-2480

33. Gicquel C, Gaston V, Mandelbaum J, Siffroi JP, Flahault A, Le Bouc Y (2003) In vitro fertilization may increase the risk of Beckwith-Wiedemann syndrome related to the abnormal imprinting of the KCN1OT gene. Am J Hum Genet 72: 1338-1341

34. Maher ER et al (2003) Beckwith-Wiedemann syndrome and assisted reproduction technology (ART). J Med Genet 40: 62-64

35. Sutcliffe AG et al (2006) Assisted reproductive therapies and imprinting disorders-a preliminary British survey. Hum Reprod 21: 1009-1011

36. Ludwig M, Katalinic A, Gross S, Sutcliffe A, Varon R, Horsthemke B (2005) Increased prevalence of imprinting defects in patients with Angelman syndrome born to subfertile couples. J Med Genet 42: 289-291
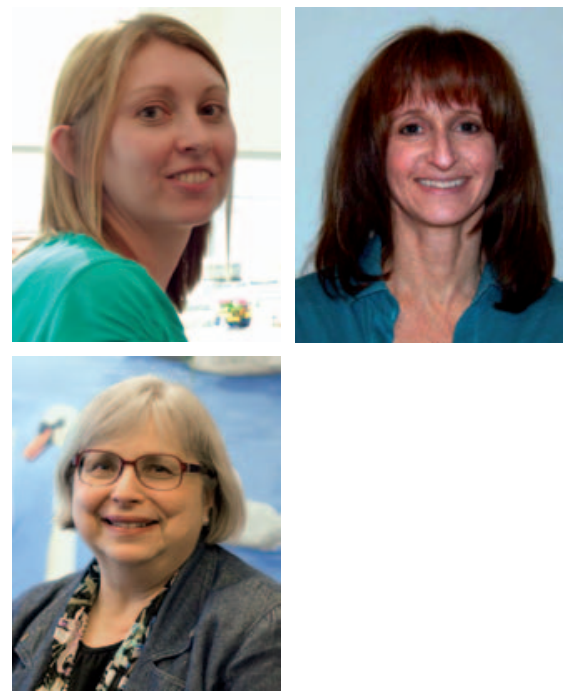

Daria Grafodatskaya, Cheryl Cytrynbaum and Rosanna Weksberg are at The Hospital for Sick Children, Toronto University in Toronto, Canada.

E-mail:rweksb@sickkids.ca

EMBO reports (2013) 14, 129-135; published online 22 January 2013; doi:10.1038/embor.2012.222 